\title{
Internet Survey of Japanese Patients With Chronic Constipation: Focus on Correlations Between Sleep Quality, Symptom Severity, and Quality of Life
}

\begin{abstract}
Sayuri Yamamoto, ${ }^{1 *}$ Yurika Kawamura, ${ }^{1}$ Kazuhiro Yamamoto, ${ }^{1}$ Yoshiharu Yamaguchi, ${ }^{1}$ Yasuhiro Tamura, ${ }^{1}$ Shinya Izawa, Hiroaki Nakagawa, ${ }^{2}$ Yoshinori Wakita, ${ }^{2}$ Yasutaka Hijikata, ${ }^{1}$ Masahide Ebi, ${ }^{1}$ Yasushi Funaki, ${ }^{1}$ Wataru Ohashi, ${ }^{3}$ Naotaka Ogasawara, ${ }^{1}$ Makoto Sasaki, ${ }^{1}$ Masato Maekawa, ${ }^{2}$ and Kunio Kasugai ${ }^{1}$

Divisions of ${ }^{1}$ Gastroenterology and ${ }^{2}$ General Medicine, Aichi Medical University School of Medicine, Yazakokarimata, Nagakute, Aichi, Japan; and ${ }^{3}$ Division of Biostatistics, Clinical Research Center, Aichi Medical University School of Medicine, Yazakokarimata, Nagakute, Aichi, Japan
\end{abstract}

\section{Background/Aims}

Chronic constipation and lifestyle factors can affect sleep quality. We evaluated the relationship between chronic constipation and sleep in the Japanese population.

\section{Methods}

This cross-sectional internet-based survey included 3000 subjects with constipation, classified according to sleep status (good/poor). Primary endpoints were Bristol stool form scale (BSFS) score and correlations between sleep disorder criteria of the Pittsburgh Sleep Quality Index (PSQI) and sleep status (good/poor sleep). Secondary endpoints included correlations between quality of life (QOL) and mood, medical, lifestyle, and sleep factors.

\section{Results}

The proportion of participants with BSFS category 4 (normal stool) was significantly higher in the good sleep group $(P<0.001)$. Sleep disturbance $(P<0.05)$, sleep quality, and duration, use of hypnotic medication, and daytime dysfunction of PSQI (all $P<0.001)$ significantly correlated with poor sleep. In the poor sleep group, QOL was significantly worse and anxiety and depression levels were significantly higher (all $P<0.001$ ) compared with the good sleep group. Anemia and smoking (both $P<0.05$ ), recent body weight increases, and poor eating habits (all $P<0.001$ ) were significantly higher in the poor sleep group. Male sex, onset associated with change in frequency of stools, sensation of incomplete evacuation for at least $25 \%$ of defecations, and manual maneuvers to facilitate at least $25 \%$ of defecations correlated with poor sleep.

\section{Conclusions}

Subjects with constipation and poor sleep experienced severe symptoms and had poor QOL. These data support the need for a multifocal treatment approach, including lifestyle advice and pharmacotherapy.

(J Neurogastroenterol Motil 2021;27:602-611)

Key Words

Constipation; Irritable bowel syndrome; Quality of life; Sleep disorders

Received: June 16, 2020 Revised: September 14, 2020 Accepted: February 18, 2021

(c) This is an Open Access article distributed under the terms of the Creative Commons Attribution Non-Commercial License (http://creativecommons. org/licenses/by-nc/4.0) which permits unrestricted non-commercial use, distribution, and reproduction in any medium, provided the original work is properly cited.

*Correspondence: Sayuri Yamamoto, MD, PhD

Division of Gastroenterology, Aichi Medical University School of Medicine, 1-1 Yazakokarimata, Nagakute, Aichi 480-1195, Japan Tel: +81-561-62-3311, Fax: +81-561-62-1508, E-mail: yuri3337@aichi-med-u.ac.jp 


\section{Introduction}

Functional gastrointestinal disorders (FGID) are commonly diagnosed in gastroenterology practice ${ }^{1}$ and are characterized by persistent and recurring gastrointestinal (GI) symptoms. Currently recognized FGID according to the Rome IV criteria include irritable bowel syndrome (IBS), functional constipation (FC) or diarrhea, functional vomiting, functional dyspepsia, and functional abdominal pain. ${ }^{1,2}$

Chronic constipation, which may present as reduced bowel movements, straining, hard stools, and/or sensations of blockage or incomplete defecation over a period of several months, ${ }^{1}$ is a disorder including constipation-predominant IBS (IBS-C) and FC, according to both Japanese guidelines (Chronic constipation medical care guideline 2017: https://www.nankodo.co.jp/g/g9784524255757/) and the Rome IV criteria. ${ }^{1}$ The prevalence of chronic constipation in Japan is estimated to be 6.1-28.0\%, depending on the assessment scale used, ${ }^{3,4}$ indicating a significant medical issue with a large socioeconomic burden.

Although studies in Western populations have indicated that constipation significantly reduces quality of life (QOL), ${ }^{5-7}$ there are few analogous data available from the Japanese population. In addition to decreased QOL, there is a well-documented bidirectional link between chronic constipation and sleep dysfunction: poor sleep may affect bowel function, ${ }^{8,9}$ and constipation may affect sleep parameters. ${ }^{10,11}$ Furthermore, lifestyle is known to affect both constipation $^{12,13}$ and sleep, ${ }^{14,15}$ but to date, there have been no studies examining the associations between these 3 factors. We hypothesized that constipation and sleep disorders are linked with each other and with lifestyle factors. Using data from a previously reported internetbased survey that investigated the actual situation of constipation in Japan, ${ }^{16}$ we investigated the relationship between chronic constipation, sleep, and lifestyle factors in the Japanese population.

\section{Materials and Methods}

\section{Study Design and Participants}

This was an internet-based survey conducted in Japan, registered at the University Hospital Medical Information Network with the identification number UMIN000024334. Full details of the survey inclusion/exclusion criteria have been published. ${ }^{16}$ In brief, adults with constipation awareness (aged 20 years to 69 years) whose identity had been confirmed by the market research firm, Rakuten Research, Inc (present: Rakuten Insight, Inc), were eligible for participation, regardless of medication usage and hospitalization status. Exclusion criteria were a presence or history of abdominal surgery (other than appendectomy), small and large intestinal diseases (such as inflammatory bowel disease, ie, ulcerative colitis or Crohn's disease), or bowel cancer or other cancers; a history of gastric/intestinal disease (such as gastric or duodenal ulcer, hemorrhoids, diverticulitis, or diverticulum); pregnancy; secondary constipation (due to a history of cerebral infarction, neurological disease, chronic obstructive lung disease, hepatic disease, or renal disease); drug-induced constipation (resulting from use of oral antidiabetic agents or insulin to treat diabetes, antihypertensive agents to treat hypertension, chalybeate [mineral spring water], hypnotics, sedatives, or antipsychotic agents); and inability to complete the survey instructions.

Between October 8, 2016 and October 11, 2016, a preliminary internet questionnaire from Rakuten Insight, Inc was completed by 10000 consenting Japanese adults via the Rakuten portal site. This presurvey was conducted to affirm the age, sex, and background details of each of the 10000 participants, including their prior medical history, presence of exclusion criteria, and their level of awareness of constipation. After eliminating participants who met the exclusion criteria or who did not provide sufficient information for analysis, 9523 participants remained eligible. Of these, based on responses to the question "Do you think you experience constipation," with answers on a 5-point Likert scale ranging from strongly disagree to strongly agree, 4908 panelists strongly or moderately agreed that they experienced constipation (Fig. 1), and were considered suitable to progress to the full survey.

To match Japanese demographic distributions of prefectural region, sex and age, based on data from the Statistics Bureau of the Ministry of Internal Affairs and Communications (October 1, 2014, http://www.stat.go.jp/data/jinsui/2014np/), 3000 people from the eligible group of 4908 were randomly selected by Rakuten to receive the full survey, using a proprietary allocation/systematic sampling method. All 3000 individuals gave informed consent for participation and were enrolled in the full internet survey; all successfully completed the survey (ie, there were no dropouts). Of these 3000 participants with constipation, 262 met the Rome III diagnostic criteria for functional constipation, as previously reported. ${ }^{16}$ The Japanese Health Practice Index was used to evaluate lifestyle parameters (effective life-style related disease guiding system using Japanese Health Practice Index: https://drive.google.com/file/d/1z LVHUNVsIVIfs4lBcbr4qFPtkaIEhKhP/view). Participants who answered "Yes" to the question, "Do you sleep well?" were classi- 


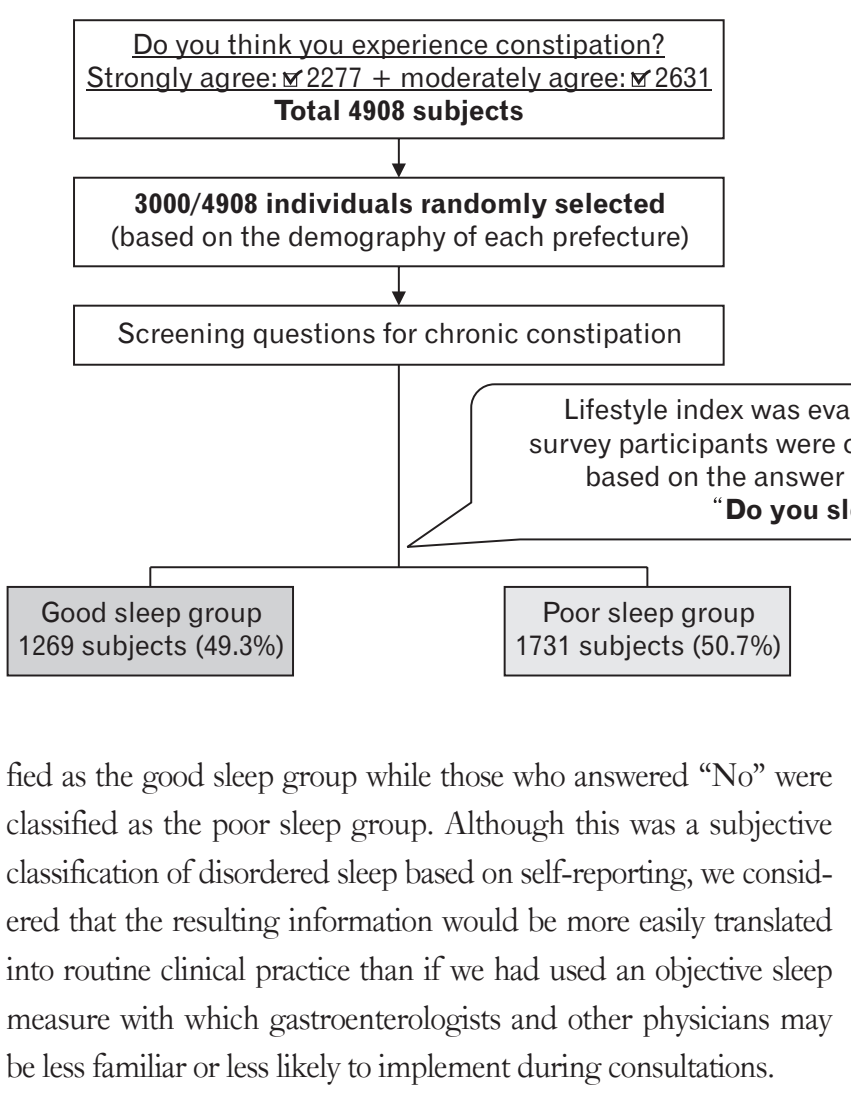

\section{Primary Endpoints}

Correlations between stool form and sleep groups were evaluated. Stools were classified (from 1 to 7 ) according to the Bristol stool form scale (BSFS), ${ }^{17}$ where types 1 and 2 indicate constipation, types 3, 4, and 5 indicate normal stools, and types 6 and 7 indicate diarrhea. Correlations between sleep disorder criteria of the Pittsburgh Sleep Quality Index (PSQI) and sleep status (good or poor) were also evaluated. ${ }^{18}$

\section{Secondary Endpoints}

Several secondary endpoints were compared between the good sleep group and the poor sleep group. We evaluated correlations between QOL and subject demographic characteristics (age, sex, body mass index, and frequency of constipation symptoms), and those between sleep and health-related QOL and mental status (depression/anxiety). For the correlations between QOL and subject demographic characteristics, the Japanese IBS severity index $(\text { IBS-SI-J) })^{19}$ and the Japanese IBS QOL scale (IBS-QOL-J) ${ }^{20}$ were used to assess QOL. The 8-dimension short-form health questionnaire (SF- 8$)^{21}$ was used to assess health-related QOL and the Hospital Anxiety and Depression Scale (HADS) $)^{22}$ was used to assess mental status.
Figure 1. Outline of the study design and participant flow. JHPI, Japanese Health Practice Index.
Items from the Rome III diagnostic questionnaire ${ }^{23-25}$ IBS$\mathrm{C}$ section and FC section, medical history, and lifestyle factors, were also compared between sleep groups. The Rome III IBS$\mathrm{C}$ criteria includes "recurrent abdominal pain or discomfort for at least 3 days per month in the last 3 months" and defecation-related items ("decreased symptoms at defecation," "onset associated with a change in the frequency of stools," and "onset associated with a change in form [appearance] of stool"). The Rome III FC criteria includes "straining during at least $25 \%$ of defecations," "lumpy or hard stools in at least $25 \%$ of defecations," "sensation of incomplete evacuation for at least $25 \%$ of defecations," "sensation of anorectal obstruction/blockage for at least $25 \%$ of defecations," "manual maneuvers to facilitate at least $25 \%$ of defecations," "fewer than 3 defecations per week," and "loose stools are rarely present without the use of laxatives."

\section{Statistical Methods}

The Clopper-Pearson method was employed to calculate two-sided $95 \% \mathrm{CI}$ by utilizing the $\mathrm{F}$ distribution. For inter-group comparisons, the Mann-Whitney $U$ test was used for continuous variables, and the $\chi^{2}$ test was used for categorical variables. In all analyses, the level of significance was set at $P<0.05$ (two-sided).

Logistic regression was conducted to extract correlation factors specifically related to good sleep and poor sleep. Univariate logistic regression was also conducted on PSQI sleep disorder criteria with sleep status (good or poor) as the objective variable; subsequently, multivariate logic regression was performed using all items with a $P$-value $<0.001$ as the explanatory variable. Age was analyzed as a continuous variable, and other factors were analyzed as categorical variables. Univariate analysis was performed on all survey items 
with sleep status as the objective variable. The likelihood ratio was then used for variable selection in multivariate analysis, using the $\chi^{2}$ test for multivariate model coefficients with $P<0.001$.

The administration of the internet survey and statistical analysis of the data were both conducted by Rakuten Insight Inc (Osaka, Japan).

\section{Ethical Considerations}

This study was approved by the institutional review board of Aichi Medical University (October 6, 2016; Approval No. 2016$\mathrm{H} 025$ ). This study was carried out in conformity with the principles of the Declaration of Helsinki and the Ethical Guidelines for Medical and Health Research Involving Human Subjects enacted by the Japanese Ministry of Education, Culture, Sports, Science and Technology and the Ministry of Health, Labour and Welfare (December 22, 2014). All authors had access to the study data and reviewed and approved the final manuscript.

\section{Results}

\section{Participant Characteristics}

Baseline characteristics are reported in Table 1. The good sleep group consisted of 595 males (46.9\%) and 674 females (53.1\%). The poor sleep group contained significantly more males than females $(P<0.003)$ : 908 males $(52.5 \%)$ and 823 females $(47.5 \%)$. The mean age \pm SD in the good sleep group and poor sleep group was $47.4 \pm 14.0$ years and $45.2 \pm 12.8$ years, respectively, with the good sleep group being significantly older $(P<0.001)$. Mean body mass index was not significantly different between groups.

\section{Primary Outcome Measures}

According to the results of the BSFS, the proportion of participants with BSFS Type 4 (normal stool) was significantly higher in the good sleep group $(P<0.001)$ (Fig. 2). Conversely, a BSFS Type 1 or 2 (lumpy stool) was associated with poor sleep $(P=0.016$ and $P=0.002$, respectively).

A logistic regression analysis was performed to extract factors characteristic of good or poor sleep using sleep disorder criteria from the PSQI with sleep status as the objective variable (Table 2). In univariate analyses, all variables with the exception of sleep efficiency had a significant impact on sleep $(P<0.001)$. Further analysis of significant variables in a multivariate analysis found that sleep quality $(P<0.001)$, duration $(P<0.001)$, sleep disturbance $(P=$ $0.050)$, use of hypnotic medication $(P=0.005)$, and daytime dysfunction $(P<0.001)$ correlated with poor sleep.

\section{Secondary Outcome Measures}

The IBS-SI-J score was significantly higher in the poor sleep group $(P<0.001)$. The proportion of participants with a moder-

Table 1. Comparison of Background Factors Between Good Sleep and Poor Sleep Groups

\begin{tabular}{lccr}
\hline $\begin{array}{c}\text { Background } \\
\text { factors }\end{array}$ & $\begin{array}{c}\text { Good sleep group } \\
(\mathrm{n}=1269)\end{array}$ & $\begin{array}{c}\text { Poor sleep group } \\
(\mathrm{n}=1731)\end{array}$ & P-value \\
\hline Males & $595(46.9)$ & $908(52.5)$ & $0.003^{\mathrm{a}}$ \\
Females & $674(53.1)$ & $823(47.5)$ & \\
Age $(\mathrm{yr})$ & $47.4 \pm 14.0$ & $45.2 \pm 12.8$ & $<0.001^{\mathrm{b}}$ \\
BMI $\left(\mathrm{kg} / \mathrm{m}^{2}\right)$ & $21.6 \pm 3.5$ & $21.6 \pm 3.6$ & $0.912^{\mathrm{b}}$ \\
${ }^{\mathrm{a}} \chi^{2}$ test. & & \\
${ }^{\mathrm{b}}$ Mann-Whitney $U$ test. \\
BMI, body mass index. \\
\multicolumn{3}{l}{ Data are presented as $\mathrm{n}(\%)$ or mean $\pm \mathrm{SD}}$.
\end{tabular}

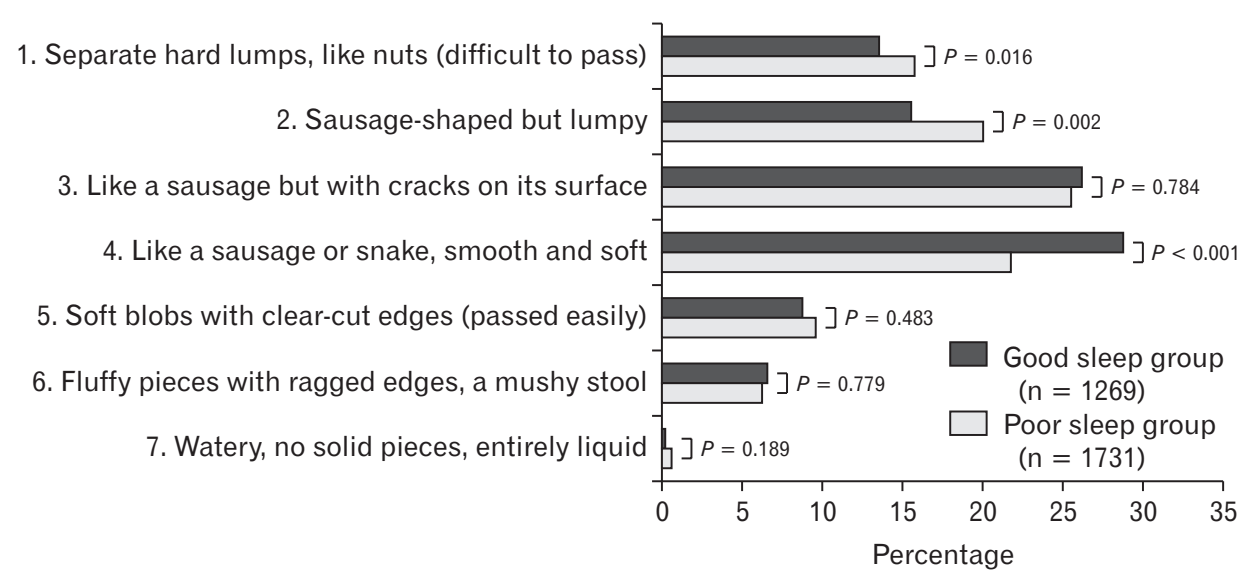

Figure 2. Comparison of Bristol stool form scale scores between the good sleep and the poor sleep groups. $P$-values were calculated using the $\chi^{2}$ test. 
Table 2. Logistic Regression Analysis of Correlations Between Sleep Disorder Criteria of the Pittsburgh Sleep Quality Index and Sleep Status (Good/Poor) in Constipated Participants ${ }^{\mathrm{a}}$

\begin{tabular}{|c|c|c|c|c|c|c|}
\hline \multirow{2}{*}{ PSQI sleep disorder criteria } & \multicolumn{3}{|c|}{ Univariate } & \multicolumn{3}{|c|}{ Multivariate } \\
\hline & OR & $95 \% \mathrm{CI}$ & $P$-value ${ }^{c}$ & OR & $95 \% \mathrm{CI}$ & $P$-value ${ }^{\mathrm{b}}$ \\
\hline Sleep quality & 0.111 & $0.094-0.130$ & $<0.001$ & 0.131 & $0.110-0.158$ & $<0.001$ \\
\hline Sleep latency & 0.407 & $0.346-0.477$ & $<0.001$ & 1.008 & $0.814-1.249$ & 0.940 \\
\hline Sleep duration & 0.296 & $0.255-0.344$ & $<0.001$ & 0.339 & $0.286-0.401$ & $<0.001$ \\
\hline Sleep efficiency ${ }^{c}$ & 1.012 & $0.938-1.093$ & 0.755 & - & - & - \\
\hline Sleep disturbance & 0.447 & $0.384-0.522$ & $<0.001$ & 0.813 & $0.661-1.000$ & 0.050 \\
\hline Use of hypnotic medication & 0.845 & $0.782-0.913$ & $<0.001$ & 1.156 & $1.044-1.280$ & 0.005 \\
\hline Daytime dysfunction & 0.453 & $0.411-0.500$ & $<0.001$ & 0.698 & $0.617-0.789$ & $<0.001$ \\
\hline
\end{tabular}

${ }^{a}$ In order to extract correlation factors that are related to good sleep and poor sleep, a univariate and multivariate logistic regression analysis was performed for survey items related to sleep (Pittsburgh Sleep Quality Index [PSQI]) with sleep status (good or poor) as the objective variable. Variable selection was used in a multivariate logistic regression analysis to determine significant variables in the univariate analysis.

$\chi^{2}$ test.

${ }^{c} \mathrm{~N}=3000$ for all, with the exception of sleep efficiency, where $\mathrm{n}=2986$.

Table 3. Comparison of Japanese Irritable Bowel Syndrome Severity Index, Japanese Irritable Bowel Syndrome Quality of Life Scale, and Hospital Anxiety and Depression Scale Between Good Sleep and Poor Sleep Groups

\begin{tabular}{lccc}
\hline \multicolumn{1}{c}{ Factors } & $\begin{array}{c}\text { Good sleep } \\
\text { group } \\
(\mathrm{n}=1269)\end{array}$ & $\begin{array}{c}\text { Poor sleep } \\
\text { group } \\
(\mathrm{n}=1731)\end{array}$ & P-value \\
\hline $\begin{array}{l}\text { IBS-SI-J score } \\
\text { Subjects with IBS-SI-J score } \\
\text { of moderate or higher }\end{array}$ & $337(26.9 \pm 76.4$ & $159.8 \pm 87.8$ & $<0.001^{\mathrm{c}}$ \\
IBS-QOL-J score & $83.2 \pm 18.2$ & $76.4 \pm 21.4$ & $<0.001^{\mathrm{c}}$ \\
HADS-anxiety & $508(40.0)$ & $1041(60.1)$ & $<0.001^{\mathrm{d}}$ \\
$\begin{array}{l}\text { Positive } \\
\text { Score }\end{array}$ & $5.8 \pm 3.8$ & $8.0 \pm 4.3$ & $<0.001^{\mathrm{c}}$ \\
HADS-depression & $726(57.2)$ & $1277(73.8)$ & $<0.001^{\mathrm{d}}$ \\
$\begin{array}{l}\text { Positive } \\
\text { Score }\end{array}$ & $8.2 \pm 3.8$ & $9.6 \pm 3.6$ & $<0.001^{\mathrm{c}}$ \\
\hline
\end{tabular}

${ }^{\mathrm{a}}$ Moderate: 175-300.

${ }^{\mathrm{b}}$ Positive: $\geq 7.0$.

${ }^{\mathrm{c}}$ Mann-Whitney $U$ test.

${ }^{\mathrm{d}} \chi^{2}$ test.

IBS-SI-J, Japanese irritable bowel syndrome severity index; IBS-QOL-J, Japanese irritable bowel syndrome quality of life; HADS, Hospital Anxiety and Depression Scale.

Data are presented as $\mathrm{n}(\%)$ or mean $\pm \mathrm{SD}$.

ate degree of severity or higher was significantly greater in the poor sleep group $(P<0.001)$. The mean IBS-QOL-J was significantly lower in the poor sleep group $(P<0.001)$ (Table 3$)$. The poor sleep group also had significantly lower scores for all SF-8 items $(P<0.001)$ (Fig. 3A).

Based on the HADS scores, the poor sleep group had significantly higher levels of both anxiety and depression $(P<0.001$, each). The positive rate of 7.0 or higher was also significantly higher in the poor sleep group $(P<0.001)$ (Table 3$)$.

Rome III diagnostic criteria, including straining during at least $25 \%$ of defecations $(P<0.001)$, lumpy or hard lumps in at least $25 \%$ of defecations $(P=0.002)$, sensation of incomplete evacuation for at least $25 \%$ of defecations $(P<0.001)$, sensation of anorectal obstruction/blockage for at least $25 \%$ of defecations $(P<$ 0.001 ), manual maneuvers to facilitate at least $25 \%$ of defecations $(P=0.002)$, recurrent abdominal pain or discomfort at least 3 days per month in the last 3 months $(P<0.001)$, onset associated with a change in frequency of stools $(P<0.001)$, and onset associated with a change in form (appearance) of stool $(P<0.001)$ were significantly higher in the poor sleep group (Fig. 3B).

Based on the assessment of medical history and lifestyle factors, anemia $(P=0.041)$, smoking $(P=0.027)$, increase of at least $3 \mathrm{~kg}$ of body weight since last year $(P<0.001)$, supper within 2 hours prior to sleep $(P<0.001)$, eating a bedtime snack $(P<0.001)$, and not eating breakfast $(P<0.001)$ were all significantly higher in the poor sleep group. Conversely, moderate exercise for $\geq 30 \mathrm{~min}$ utes at least twice per week for $\geq 1$ year $(P=0.017)$ and exercise equivalent to walking for $\geq 1$ hour a day $(P=0.034)$ were significantly higher in the good sleep group (Fig. 3C).

Based on multivariate likelihood analysis of subject factors, variables related to poor sleep in this population of individuals with constipation included male sex, onset associated with a change in frequency of stool, sensation of incomplete evacuation for at least $25 \%$ of defecations, and manual maneuvers to facilitate at least $25 \%$ of defecations. Variables related to good sleep were older age and a BSFS score of 3-5 (Table 4). 


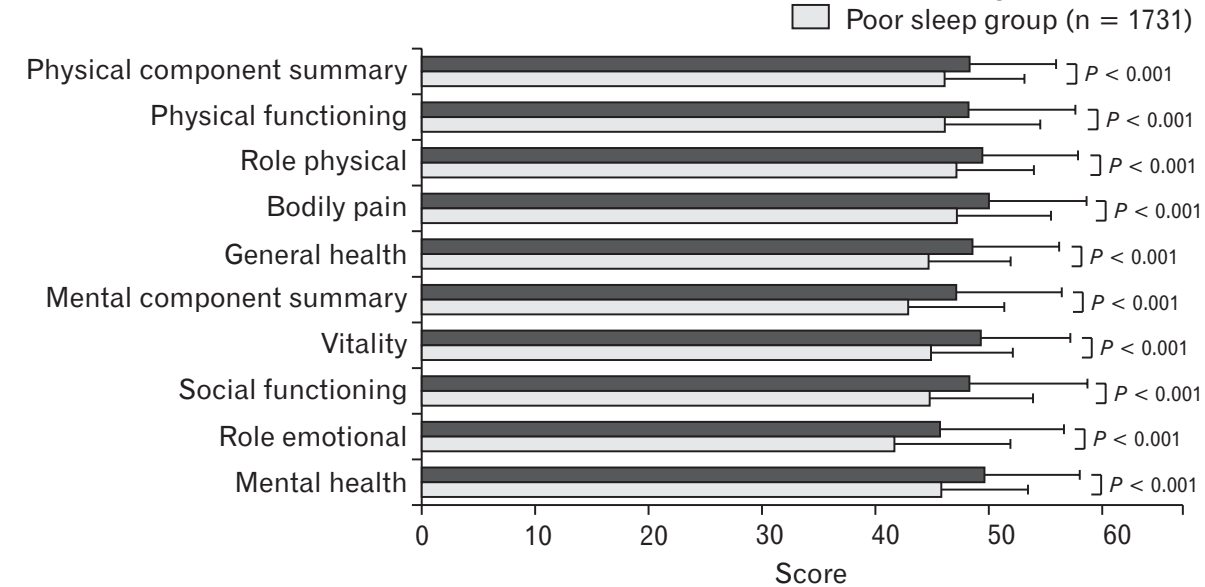

B Straining during at least $25 \%$ of defecations Lumpy or hard stools in
at least $25 \%$ of defecations Sensation of incomplete evacuation for at least $25 \%$ of defecations

Sensation of anorectal obstruction/blockage for at least $25 \%$ of defecations

Manual maneuvers to facilitate at least $25 \%$ of defecations

Fewer than 3 defecations per week

Loose stools are rarely present without the use of laxatives Recurrent abdominal pain or discomfort at least 3 days per month in the last 3 mo

Improvement with defecation

Onset associated with a change in frequency of stools Onset associated with a change
in form (appearance) of stool

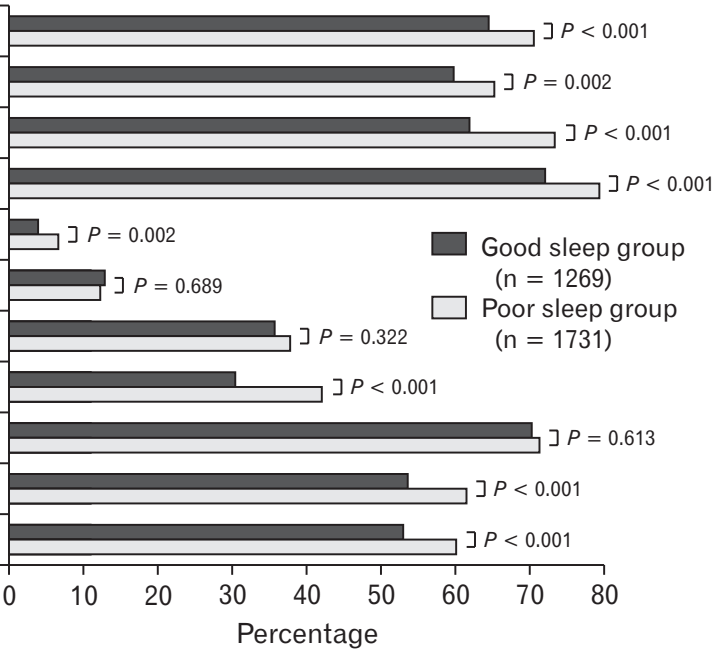

C

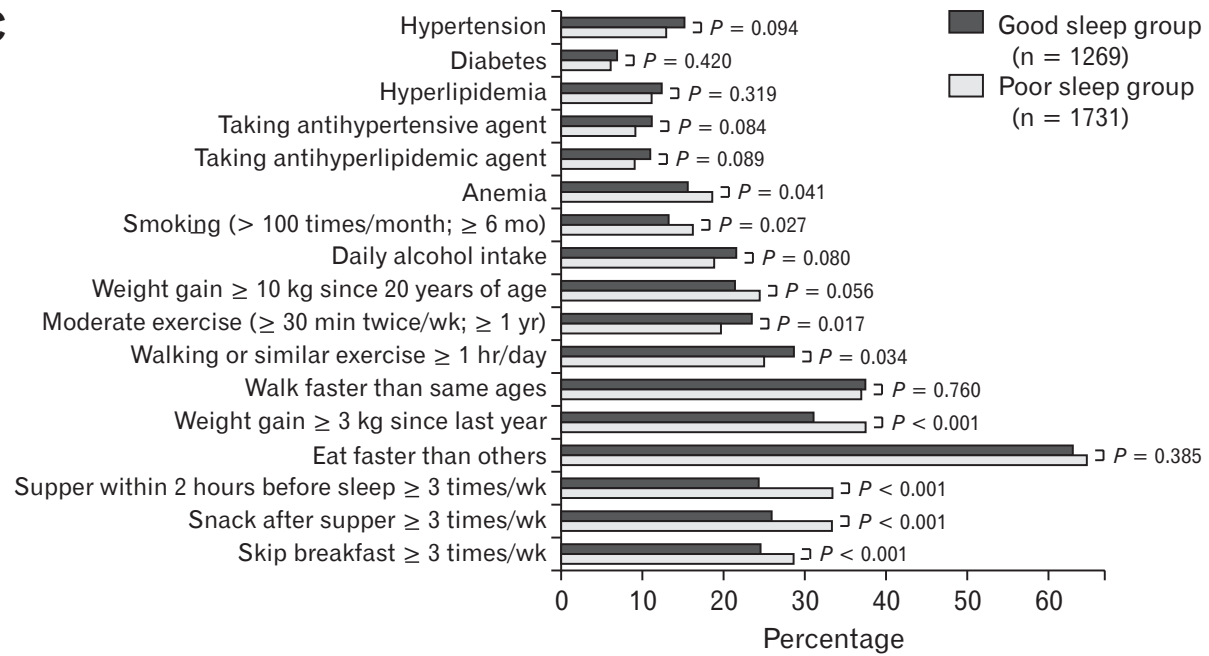

Figure 3. Comparison of (A) Short Form-8 Health Survey (SF-8) ( $P$-values were calculated using the Mann-Whitney $U$ test), (B) Rome III criteria $\left(P\right.$-values were calculated using the $\chi^{2}$ test), and $(\mathrm{C})$ lifestyle factors $\left(P\right.$-values were calculated using the $\chi^{2}$ test $)$ between the good sleep and the poor sleep groups. 
Table 4. Logistic Regression Analysis for Sleep Quality in Japanese Individuals With Constipation ${ }^{\text {a }}$

\begin{tabular}{|c|c|c|c|c|c|c|}
\hline \multirow{2}{*}{ Variables } & \multicolumn{3}{|c|}{ Univariate } & \multicolumn{3}{|c|}{ Multivariate } \\
\hline & OR & $95 \% \mathrm{CI}$ & $P$-value ${ }^{\mathrm{b}}$ & OR & $95 \% \mathrm{CI}$ & $P$-value \\
\hline Sex (male vs female) & 0.800 & $0.692-0.925$ & 0.003 & 0.783 & $0.675-0.907$ & 0.001 \\
\hline Age (1-year increment) & 1.012 & $1.007-1.018$ & $<0.001$ & 1.012 & $1.006-1.017$ & $<0.001$ \\
\hline Strong awareness of constipation (yes vs no) & 0.819 & $0.708-0.948$ & 0.007 & - & - & - \\
\hline Defecation improves abdominal pain and gastrointestinal symptoms (yes vs no) & 0.957 & $0.816-1.122$ & 0.585 & - & - & - \\
\hline Onset associated with a change in frequency of stools (yes vs no) & 0.728 & $0.629-0.843$ & $<0.001$ & 0.832 & $0.714-0.968$ & 0.018 \\
\hline Onset associated with a change in form (appearance) of stool (yes vs no) & 0.753 & $0.650-0.871$ & $<0.001$ & - & - & - \\
\hline Straining during at least $25 \%$ of defecations ${ }^{c}$ (yes vs no) & 0.762 & $0.653-0.890$ & $<0.001$ & - & - & - \\
\hline Lumpy or hard stools in at least $25 \%$ of defecations ${ }^{c}$ (yes vs no) & 0.790 & $0.680-0.918$ & 0.002 & - & - & - \\
\hline Sensation of incomplete evacuation for at least $25 \%$ of defecations ${ }^{c}$ (yes vs no) & 0.587 & $0.503-0.686$ & $<0.001$ & 0.627 & $0.533-0.737$ & $<0.001$ \\
\hline $\begin{array}{l}\text { Sensation of anorectal obstruction/blockage for at least } 25 \% \text { of defecations }{ }^{c} \\
\text { (yes vs no) }\end{array}$ & 0.672 & $0.567-0.796$ & $<0.001$ & - & - & - \\
\hline Manual maneuvers to facilitate at least $25 \%$ of defecations $^{\mathrm{d}}$ (yes vs no) & 0.575 & $0.408-0.811$ & 0.002 & 0.696 & $0.491-0.988$ & 0.043 \\
\hline Loose stools are rarely present without the use of laxatives (yes vs no) & 0.924 & $0.795-1.074$ & 0.304 & - & - & - \\
\hline \multicolumn{7}{|l|}{ Bristol stool form scale score } \\
\hline $1-2$ or $6-7{\text { (abnormal })^{\mathrm{d}}}$ & 1.000 & Reference & - & 1.000 & Reference & - \\
\hline $3-5{\text { (normal })^{\mathrm{d}}}$ & 1.329 & $1.145-1.542$ & $<0.001$ & 1.217 & $1.044-1.419$ & 0.012 \\
\hline
\end{tabular}

${ }^{\mathrm{a}}$ Univariate outcomes were obtained for all survey items related to constipation symptoms with sleep status (good or poor) as the objective variable. Likelihood ratio was used for variable selection in multivariate analysis.

${ }^{\mathrm{b}} \chi^{2}$ test.

${ }^{\mathrm{c}}$ Rome III criteria.

${ }^{\mathrm{d}} \mathrm{N}=3000$ for all, with the exception of Bristol stool form scale score, where $\mathrm{n}=1200$ for abnormal scores and $\mathrm{n}=1800$ for normal scores.

\section{Discussion}

Chronic constipation can have an extremely negative impact on patient well-being, ${ }^{26}$ with significant impairment of $\mathrm{QOL}^{5-7}$ and effects on sleep ${ }^{10,11}$ and mental health. ${ }^{10,27}$ Despite this, there are few published reports on the relationship between constipation and sleep in Japanese patients, and the level of scientific consideration is low.

In the present study, we investigated the differences between individuals reporting good sleep and those reporting poor sleep among 3000 Japanese survey participants with constipation. Around half of the study participants indicated that they had problems with sleep. This result is in line with previous survey data in the United States, examining the link between constipation and sleep, in which sleep scores were found to correlate significantly with GI symptom scores $(P<0.001){ }^{11}$ Similarly, a previous large-scale internet survey in Japan reported that patients with any FGID had significantly less sleep compared with control subjects $(P<0.01){ }^{28}$ The sleep duration of patients with IBS has also been shown to be relatively short, with a significant correlation reported between the quality of overnight sleep and morning IBS symptoms $(P<0.001){ }^{29}$

The relationship between constipation and sleep may poten- tially be explained by converging circadian and colonic motility rhythms, ${ }^{30,31}$ and it is well known that GI symptoms occur at a higher frequency among people with disrupted biological rhythms, such as shift workers and travelers across time zones. ${ }^{32}$ Importantly, this association is bidirectional: abnormal GI movements can be the cause of rapid eye movement sleep disorder ${ }^{33}$ and, conversely, GI disorders such as IBS can occur secondary to insomnia. ${ }^{34}$

In the present study, participants with constipation were classified according to their perception of good or poor sleep and compared by means of multiple parameters, including the BSFS, PSQI, QOL scales, HADS, the Rome III questionnaire, medical history, and lifestyle habits. This is the first such study to use these parameters to evaluate the associations between sleep, constipation, and lifestyle. A key finding of this analysis was that the proportion of participants with normal stools (BSFS type 4) was significantly lower in the poor sleep group compared with the good sleep group. Based on the results of the multivariate analysis, it can be inferred that maintaining a BSFS score between 3 and 5 (defined as normal stool) may contribute to better sleep among patients with constipation. Unfortunately, it was not possible to find any previous reports that evaluated the stool form in the specific context of sleep. However, a recent publication reported that BSFS type 4 (normal 
stool form) is important for QOL improvement among patients suffering from constipation. ${ }^{35}$ Thus, the clinical meaning of these results remains to be determined and should be further investigated. Nonetheless, we can infer that when patients with constipation are reporting sleep difficulties, it is key that clinicians manage their constipation symptoms, in order to improve sleep quality.

In logistic regression analysis using the sleep disorder criteria of the PSQI, we noted that the odds ratios were remarkably low in variables related to the quality of sleep (sleep quality and sleep duration). Conversely, the odds ratio for 'use of hypnotic medication' was $>1$. These data indicate that patients who successfully use hypnotic medication have a better quality of sleep. However, we should also caution that the use of hypnotic medication must be undertaken with care due to the potential associated risks of addiction, dementia, falls, and depression. ${ }^{36,37}$

We also noted that the IBS-SI-J score and IBS-QOL-J were significantly worse in the poor sleep group compared with the good sleep group. All SF-8 dimension scores were lower in both groups than the Japanese national average (SF-8 health survey. http:/www. qualitest.jp/qol/sf8.html) but were significantly reduced in the poor sleep group compared with the good sleep group. Moreover, levels of anxiety and depression were significantly higher in the poor sleep group. Finally, the results of the Rome III FC criteria questionnaire are of particular interest as strong associations with sleep were shown for "sensation of incomplete evacuation" and "manual maneuvers to facilitate defecation." Overall, the results obtained in our study are very similar to those obtained in an analysis of 126 adult Chinese patients with chronic constipation, ${ }^{9}$ in which individuals with a sleep disorder (defined according to the PSQI) had elevated rates of incomplete defecation, blockage, and constipation symptoms compared with individuals with normal sleep. Poor sleep in the Chinese analysis was also associated with significantly higher levels of worry and anxiety, and sleep disorders, depression, and anxiety were all found to be positively correlated with constipation severity. Interestingly, however, for patients reporting fewer than 3 defecations per week in our survey, there was no significant difference between sleep groups, suggesting that a low frequency of defecation is not sufficient in itself to influence levels of stress or mental health and, thus, negatively impact sleep.

According to a Japanese national survey of lifestyle and health (The 2013 lifestyle survey: https://www.mhlw.go.jp/toukei/saikin/ hw/k-tyosa/k-tyosa13/dl/16.pdf), the prevalence of constipation in Japan is around $2-5 \%$, while other published data suggest a higher prevalence of 6-28\%. ${ }^{3,4}$ However, patients do not always perceive constipation as a disease, physicians do not always attach the ap- propriate significance to the condition, and many sufferers use over the counter medication and supplements in an attempt to control their symptoms. ${ }^{3,38}$ These results are similar to those obtained in Western populations ${ }^{39}$ and suggest that new management pathways and more efficacious therapies are needed to reduce symptoms, improve patient satisfaction, and decrease the socioeconomic burden of chronic constipation and other FGIDs.

The limitations of this analysis stem primarily from the risk of obtaining unreliable data due to the fact that the study was based on an internet survey. However, attempts were made to overcome this limitation by using registered respondents whose identity had been confirmed by a research company. Furthermore, the participant pool was designed to match the profile of the general Japanese population (Summary report of comprehensive survey of living conditions 2017: https://www.mhlw.go.jp/toukei/saikin/hw/k-tyosa/k-tyosa17/ $\mathrm{dl} / 10$.pdf), allowing extrapolation of the data to real-world individuals with chronic constipation, and the data were obtained directly from the subjects themselves without the risk of bias from health care personnel; both of these factors enhance the potential reliability and usability of the resultant data. Finally, although it is clear that additional factors including medical history and lifestyle can also influence sleep quality, we did not attempt to include these in our multivariate analysis, preferring instead to focus on the influence of constipation and defecation status on poor sleep, plus a manageable number of factors which could clearly be confounding, such as age and sex.

In summary, our study data support the hypothesis that constipation and sleep disorders are inextricably linked with each other and with lifestyle factors. As such, multifactorial treatment strategies focusing on each of these parameters are needed to improve levels of well-being and QOL in affected individuals.

Acknowledgements: We wish to sincerely thank Hideyuki Mishima and Kenta Murotani for performing the statistical analysis, and we would also like to thank Rakuten Insight Inc (Osaka, Japan) for performing the internet survey.

Financial support: This study was funded by EA Pharma Co, Ltd (Tokyo, Japan). We would also like to thank Sally-Anne Mitchell, $\mathrm{PhD}$, of Edanz (www.edanz.com) for providing medical writing support, which was funded by EA Pharma Co, Ltd (Tokyo, Japan), in accordance with Good Publication Practice (GPP3) guidelines.

Conflicts of interest: Kunio Kasugai reports scholarship donations from AstraZeneca and Daiichi Sankyo Co, Ltd, and research 
funding from EA Pharma Co, Ltd (Tokyo, Japan). EA Pharma $\mathrm{Co}$, Ltd was not involved in designing the study protocol, implementation of the study, analysis of data, or publication of this report. The remaining authors declare no conflicts.

Author contributions: Sayuri Yamamoto was responsible for the study concept and design, arranging study funding, study investigation and methodology, project administration, and manuscript review and editing; Yurika Kawamura was responsible for the study concept and data curation; Kazuhiro Yamamoto was responsible for data curation and formal analysis of the data; Yoshiharu Yamaguchi, Yasuhiro Tamura, and Shinya Izawa were responsible for arranging study funding, project administration, and data validation; Hiroaki Nakagawa was responsible for arranging study funding and the software used for data analysis; Yoshinori Wakita was responsible for study investigation, project administration, data validation, and writing the original draft of the manuscript; Yasutaka Hijikata was responsible for study methodology, project administration, and resource acquisition; Masahide Ebi was responsible for project administration, and resource acquisition; Yasushi Funaki was responsible for project administration, and the software used for data analysis; Wataru Ohashi was responsible for the formal analysis of the data; Naotaka Ogasawara was responsible for data visualization; Makoto Sasaki was responsible for writing the original draft of the manuscript; Masato Maekawa was responsible for project administration and supervision; and Kunio Kasugai was responsible for the study concept, arranging study funding, and manuscript review and editing. All authors provided final approval for manuscript submission and agreed to be accountable for the accuracy and integrity of the data.

\section{References}

1. Drossman DA. Functional gastrointestinal disorders: history, pathophysiology, clinical features and Rome IV. Gastroenterology 2016;150:12621279, e2.

2. Stanghellini V. Functional dyspepsia and irritable bowel syndrome: beyond Rome IV. Dig Dis 2017;35(suppl 1):14-17.

3. Tamura A, Tomita T, Oshima T, et al. Prevalence and self-recognition of chronic constipation: results of an internet survey. J Neurogastroenterol Motil 2016;22:677-685.

4. Ono M, Kato M, Miyamoto S, et al. Multicenter observational study on functional bowel disorders diagnosed using Rome III diagnostic criteria in Japan. J Gastroenterol 2018;53:916-923.

5. Brochard C, Chambaz M, Ropert A, et al. Quality of life in 1870 patients with constipation and/or fecal incontinence: constipation should not be underestimated. Clin Res Hepatol Gastroenterol 2019;43:682-687.
6. Dennison C, Prasad M, Lloyd A, Bhattacharyya SK, Dhawan R, Coyne $\mathrm{K}$. The health-related quality of life and economic burden of constipation. Pharmacoeconomics 2005;23:461-476.

7. Sun SX, Dibonaventura M, Purayidathil FW, Wagner JS, Dabbous O, Mody R. Impact of chronic constipation on health-related quality of life, work productivity, and healthcare resource use: an analysis of the national health and wellness survey. Dig Dis Sci 2011;56:2688-2695.

8. Gwee KA. Disturbed sleep and disturbed bowel functions: implications for constipation in healthy individuals. J Neurogastroenterol Motil 2011;17:108-109.

9. Jiang Y, Tang YR, Xie C, Yu T, Xiong WJ, Lin L. Influence of sleep disorders on somatic symptoms, mental health, and quality of life in patients with chronic constipation. Medicine 2017;96:e6093.

10. Han SH, Lee OY, Lee YS, Kim KB, Yoon BC, Choi HS. Anxiety, depression and sleep disturbance in female constipation predominant irritable bowel syndrome. Korean J Neurogastroenterol Motil 2005;11:6671.

11. Cremonini F, Camilleri M, Zinsmeister AR, Herrick LM, Beebe T, Talley NJ. Sleep disturbances are linked to both upper and lower gastrointestinal symptoms in the general population. Neurogastroenterol Motil 2009;21:128-135.

12. Chang LL, Lin YC, Lo TC, Chen MC, Kuo HW. Understanding the lifestyle correlates with chronic constipation and self-rated health. Food Nutrition Sci 2015;6:391-398.

13. Mugie SM, Benninga MA, Di Lorenzo C. Epidemiology of constipation in children and adults: a systematic review. Best Pract Res Clin Gastroenterol 2011;25:3-18.

14. Baker FC, Wolfson AR, Lee KA. Association of sociodemographic, lifestyle, and health factors with sleep quality and daytime sleepiness in women: findings from the 2007 national sleep foundation "Sleep in America Poll”. J Womens Health 2009;18:841-849.

15. Monk TH, Reynolds CF 3rd, Buysse DJ, DeGrazia JM, Kupfer DJ. The relationship between lifestyle regularity and subjective sleep quality. Chronobiol Int 2003;20:97-107.

16. Kawamura Y, Yamamoto S, Funaki Y, et al. Internet survey on the actual situation of constipation in the Japanese population under 70 years old: focus on functional constipation and constipation-predominant irritable bowel syndrome. J Gastroenterol 2020;55:27-38.

17. Lewis SJ, Heaton KW. Stool form scale as a useful guide to intestinal transit time. Scand J Gastroenterol 1997;32:920-924.

18. Buysse DJ, Reynolds CF 3rd, Monk TH, Berman SR, Kupfer DJ. The Pittsburgh sleep quality index: a new instrument for psychiatric practice and research. Psychiatry Res 1989;28:193-213.

19. Shinozaki M, Kanazawa M, Sagami Y, et al. Validation of the Japanese version of the Rome II modular questionnaire and irritable bowel syndrome severity index. J Gastroenterol 2006;41:491-494.

20. Kanazawa M, Drossman DA, Shinozaki M, et al. Translation and validation of a Japanese version of the irritable bowel syndrome-quality of life measure (IBS-QOL-J). Biopsychosoc Med 2007;1:6.

21. Tokuda Y, Okubo T, Ohde S, et al. Assessing items on the SF-8 Japanese version for health-related quality of life: a psychometric analysis based on the nominal categories model of item response theory. Value Health 
2009;12:568-573.

22. Hatta $H$, Higashi A, Yashiro $H$, et al. [A validation of the hospital anxiety and depression scale.] Jpn J Psychosom Med 1998;38:309-315. [Japanese]

23. Kanazawa M, Nakajima S, Oshima T, et al. Validity and reliability of the Japanese version of the Rome III diagnostic questionnaire for irritable bowel syndrome and functional dyspepsia. J Neurogastroenterol Motil 2015;21:537-544.

24. Drossman DA, Corazziari E, Delvaux M, et al. Rome III: the functional gastrointestinal disorders, 3rd ed. McLean, VA: Degnon Associates 2006.

25. Longstreth GF, Thompson WG, Chey WD, Houghton LA, Mearin F, Spiller RC. Functional bowel disorders. Gastroenterology 2006;130:1480-1491.

26. Sanchez MI, Bercik P. Epidemiology and burden of chronic constipation. Can J Gastroenterol 2011;25(suppl B):11B-15B.

27. Cheng C, Chan AO, Hui WM, Lam SK. Coping strategies, illness perception, anxiety and depression of patients with idiopathic constipation: a population-based study. Aliment Pharmacol Ther 2003;18:319-326.

28. Miwa H. Life style in persons with functional gastrointestinal disorders-large-scale internet survey of lifestyle in Japan. Neurogastroenterol Motil 2012;24:464-471, e217.

29. Goldsmith G, Levin JS. Effect of sleep quality on symptoms of irritable bowel syndrome. Dig Dis Sci 1993;38:1809-1814.

30. Keller J, Gröger G, Cherian L, Günther B, Layer P. Circadian coupling between pancreatic and intestinal motility in humans. Am J Physiol Gastrointest Liver Physiol 2001;280:G273-G278.
31. Bassotti G, Iantorno G, Fiorella S, Bustos-Fernandez L, Bilder CR. Colonic motility in man: features in normal subjects and in patients with chronic idiopathic constipation. Am J Gastroenterol 1999;94:1760-1770.

32. Hoogerwerf WA. Role of clock genes in gastrointestinal motility. Am J Physiol Gastrointest Liver Physiol 2010;299:G549-G555.

33. Kumar D, Thompson PD, Wingate DL, Vesselinova-Jenkins CK, Libby G. Abnormal REM sleep in the irritable bowel syndrome. Gastroenterology 1992;103:12-17.

34. Jarrett M, Heitkemper M, Cain KC, Burr RL, Hertig V. Sleep disturbance influences gastrointestinal symptoms in women with irritable bowel syndrome. Dig Dis Sci 2000;45:952-959.

35. Ohkubo H, Yoshihara T, Misawa N, et al. Relationship between stool form and quality of life in patients with chronic constipation: an internet questionnaire survey. Digestion 2021;102:147-154.

36. Janhsen K, Roser P, Hoffmann K. The problems of long-term treatment with benzodiazepines and related substances. Dtsch Arztebl Int 2015;112:1-7.

37. Tseng LY, Huang ST, Peng LN, Chen LK, Hsiao FY. Benzodiazepines, z-hypnotics, and risk of dementia: special considerations of half-lives and concomitant use. Neurotherapeutics 2020;17:156-164.

38. Iizuka N, Hamamoto Y. Constipation and herbal medicine. Front Pharmacol 2015;6:73.

39. Quigley EMM, Horn J, Kissous-Hunt M, Crozier RA, Harris LA. Better understanding and recognition of the disconnects, experiences, and needs of patients with irritable bowel syndrome with constipation (BURDEN IBS-C) study: results of an online questionnaire. Adv Ther 2018;35:967-980. 